\title{
Special issue on emergence in human-like intelligence toward cyber- physical systems
}

\author{
Zheng $\mathrm{Xu}^{1} \cdot$ Neil Y. Yen ${ }^{2}$
}

Published online: 21 January 2019

(C) Springer-Verlag London Ltd., part of Springer Nature 2019

As the famous slogan "Connecting People" indicates, a lot of the developments in novel technologies intensify the relationship between people without necessarily enhancing technologies that are close to the nature of human beings. Examples can be easily found in recent computing paradigms, such as Cloud Computing that advances network infrastructure for data storage and resource sharing, or the Internet of Things that investigates the intelligence and awareness of objects involved in the network. From this point of view, the existing state-of-the-art solutions, in realms of artificial intelligence and/or computational intelligence, significantly differ from the human competence level in many research domains. Even though it is generally not clear whether human-like approach would show its upper-hand over existing methods, the exploration of this research path seems to be advantageous and challenging. Human-like intelligence is arguably one of the most powerful approaches for the optimization and design of complex Web, as well as its correlated issues so as to meet the requirements and growing needs of new challenging applications (and services as well). Although the state of the art behind the computational intelligence has already reached an impressive scale, there remain quite a lot of attractive research topics, with consideration of human-like or human-oriented factors, for further and promising developments.

The submitted manuscripts were reviewed by experts from both academia and industry. After two rounds of reviewing, the highest quality manuscripts were accepted for this special issue. This special issue will be published

Zheng Xu

xuzheng@shu.edu.cn

Neil Y. Yen

neilyyen@u-aizu.ac.jp

1 The Third Research Institute of the Ministry of Public Security, Shanghai, China

2 The University of Aizu, Aizu-Wakamatsu, Fukushima Prefecture 965-8580, Japan by Neural Computing and Applications as special issues. Totally, 28 papers are suggested to EiC for acceptance from 44 manuscript submissions. The selected papers are summarized as follows.

Zhang et al. [1] proposed an adaptive congestion control protocol (ACCP) which is divided into two phases to control network congestion before affecting network performance. Hou et al. [2] presented a methodology for identifying low-carbon travel block, which can be used to identify the built environment conducive to residents' lowcarbon travel. Cui et al. [3] introduced the process of material deterioration of existing reinforced concrete structures and evaluated its effects on their seismic performance by proposing the three-dimensional fragility curve based on hybrid sensing method. Cui and Li [4] represent the probabilities of the basic events by functions. The variables of the function are $n$ influencing factors on the basic events. Due to mass prestack seismic data, existing single computer environment cannot satisfy computation requirement of huge data size. Thus, an efficient and fast method is proposed by $\mathrm{Hu}$ et al. [5] to solve the inversion problem of prestack seismic big data. Song et al. [6] proposed a method named multiple-order semantic relation extraction (MOSRE), which applies for multiple orders, a conceptual expression used in formal logistics, to build semantic patterns for extracting information from hybrid unstructured texts in the open domain with deep semantic analyses. Xie and Peng [7] applied recently emerged ensemble learning methods to predict the burned area of forest fires and the occurrence of large-scale forest fires using the forest fire dataset from the University of California, Irvine machine learning repository collected from the northeastern region of Portugal. Song et al. [8] used some natural language processing ways, such as word embedding and combining semantic, to let the machine realize the content of supervision video and then focus on the civil engineering supervision video retrieval annotated by supervision engineer. Mou et al. [9] investigated the single-machine inverse scheduling problem with adjusted 
due dates (SISPAD) which has a strong background in practical industries. In the SISPAD, the parameters' values are uncertain, and the objective is to obtain the optimal schedule sequence through minimal adjusting processing parameters or the job sequence for a promising target. As the implementation of parallel in multi-machine is relatively difficult, $\mathrm{Li}$ et al. [10] put forward a recognition method based on convolution neural network algorithm, so as to improve the small batch stochastic gradient descent algorithm that is very popular in the industry. To achieve the parameters' optimization and calibration for the distributed, conceptual watershed Xinanjiang model effectively and accurately, a multi-objective artificial bee colony algorithm named RMOABC which adopts the mechanisms of regulation operator and Adaptive Grid is introduced by Huo and Liu [11]. Li et al. [12] proposed a hybrid recommendation algorithm which can improve the efficiency of traditional recommendation algorithm. The presented research introduces the whole process of user interest model and teaching resources model, which also designs and implements the personalized network teaching resources system prototype. Duan et al. [13] proposed a novel social recommendation method combined with a restricted Boltzmann machine model and trust information to improve the performance of recommendations. Zhang et al. [14] proposed a new model that introduces the concept of hybrid kernel and sample selection method based on an online learning model using a membership function. In other words, an online sequential extreme learning machine based on a hybrid kernel function (HKOS-ELM) is presented. The particle swarm algorithm that optimizes the weight values of wavelet neural network (scale factor) and threshold value (the translation factor) was developed by Yang and Chen [15] to reduce the iteration times and improve the convergence precision and rapidity so that the various parameters of wavelet neural network can be chosen adaptively. Li [16] constructed three types of single-hidden layer feed-forward neural networks (FNNs) with optimized piecewise linear activation functions and fixed weights and to present the ideal upper- and lowerbound estimations on the approximation accuracy of the FNNs, for continuous function defined on bounded intervals. The guaranteed cost control problem for mode-dependent time-delay Markov switching singular systems with norm-bounded uncertain parameters is discussed by Liu et al. [17]. A method combining median estimation with Huber $\mathrm{M}$ estimation based on robust theory is proposed by $\mathrm{Xu}$ and Xia [18] to establish prior distributions for Bayesian methods, and the posterior distribution is induced according to Bayesian methods. $\mathrm{Li}$ et al. [19] present a new method named density-distance and heuristic for identifying temporal protein complexes. Xiao et al. [20] proposed a partial fingerprint recognition algorithm based on deep learning for the recognition of partial fingerprint images. It can improve the structure of convolutional neural networks, use two kinds of loss functions for network training and feature extraction, and finally improve the recognition performance of partial fingerprint images. To realize real-time fault detection in power devices and enhance reliability of inverter circuits, Ji and Liu [21] proposed a detection method based on Park's transform algorithm and neural network. Lin et al. [22] study automatically crawls the information published by users of the MedHelp Medical Forum and then combines it with disease-related user posts obtained from Twitter. Dong et al. [23] proposed a method to evaluate the degree of emotion being motivated in continuous music videos based on asymmetry index (AsI). Zhao et al. [24] proposed a new neural network; it combines convolutional neural network $(\mathrm{CNN})$ with general regression neural network (GRNN), which is written as the CNN-GRNN. The sparse subspace clustering (SSC) algorithm is introduced by Wang et al. [25] to analyze the time series data. The SSC algorithm has a better performance on both the artificial dataset and the daily box-office data than recently developed well-known clustering algorithms such as K-means and spectral clustering algorithm. Liu et al. [26] proposed a charging demand simulation method based on the Agent-cellular automata model to describe the changes in location and the state of charge of a moving EV. Li et al. [27] used the iterative learning control algorithm to deal with generalized distributed parameter system with parabolic type described by generalized partial differential equation. Yang [28] used NetLogo simulation platform, using the overall modeling and simulation method of Multi-Agent, established the asymmetric evolutionary game simulation model which participates in the three parties, and run the model under different revenue parameters.

Acknowledgements The guest editors would like to thank Prof. John MacIntyre who is the editor in chief of Neural Computing and Applications. His help and trust is the most important thing for the success of this SI. The guest editors would like to thank the reviewers for their high-quality reviews, which provided insightful and constructive feedback to the authors of the papers.

\section{References}

1. Liu T, Zhang M, Zhu J et al (2018) ACCP: adaptive congestion control protocol in named data networking based on deep learning. Neural Comput Appl. https://doi.org/10.1007/s00521018-3408-2

2. Hou Q, Zhang X, Li B et al (2018) Identification of low-carbon travel block based on GIS hotspot analysis using spatial distribution learning algorithm. Neural Comput Appl. https://doi.org/ 10.1007/s00521-018-3447-8 
3. Cui J, Xie H, Cui P et al (2018) Seismic performance evaluation of existing RC structures based on hybrid sensing method. Neural Comput Appl. https://doi.org/10.1007/s00521-018-3448-7

4. Cui TJ, Li S (2018) Deep learning of system reliability under multi-factor influence based on space fault tree. Neural Comput Appl. https://doi.org/10.1007/s00521-018-3416-2

5. Yan X, Zhu Z, Hu C et al (2018) Spark-based intelligent parameter inversion method for prestack seismic data. Neural Comput Appl. https://doi.org/10.1007/s00521-018-3457-6

6. Song S, Sun Y, Di Q (2018) Multiple order semantic relation extraction. Neural Comput Appl. https://doi.org/10.1007/s00521018-3453-X

7. Xie Y, Peng M (2018) Forest fire forecasting using ensemble learning approaches. Neural Comput Appl. https://doi.org/10. 1007/s00521-018-3515-0

8. Wu S, Song H, Cheng G et al (2018) Civil engineering supervision video retrieval method optimization based on spectral clustering and R-tree. Neural Comput Appl. https://doi.org/10. 1007/s00521-018-3485-2

9. Mou J, Gao L, Guo Q et al (2018) Hybrid optimization algorithms by various structures for a real-world inverse scheduling problem with uncertain due-dates under single-machine shop systems. Neural Comput Appl. https://doi.org/10.1007/s00521-018-3472-7

10. Shu Y, Huang Y, Li B (2018) Design of deep learning accelerated algorithm for online recognition of industrial products defects. Neural Comput Appl. https://doi.org/10.1007/s00521-018-3511-4

11. Huo J, Liu L (2018) Application research of multi-objective Artificial Bee Colony optimization algorithm for parameters calibration of hydrological model. Neural Comput Appl. https:// doi.org/10.1007/s00521-018-3483-4

12. Li H, Li H, Zhang S et al (2018) Intelligent learning system based on personalized recommendation technology. Neural Comput Appl. https://doi.org/10.1007/s00521-018-3510-5

13. Wu X, Yuan X, Duan C et al (2018) A novel collaborative filtering algorithm of machine learning by integrating restricted Boltzmann machine and trust information. Neural Comput Appl. https://doi.org/10.1007/s00521-018-3509-y

14. Zhang S, Tan W, Wang Q et al (2018) A new method of online extreme learning machine based on hybrid kernel function. Neural Comput Appl. https://doi.org/10.1007/s00521-018-3629-4

15. Yang L, Chen H (2018) Fault diagnosis of gearbox based on RBF-PF and particle swarm optimization wavelet neural network. Neural Comput Appl. https://doi.org/10.1007/s00521-018-3525-y

16. Li FJ (2018) Constructive function approximation by neural networks with optimized activation functions and fixed weights. Neural Comput Appl. https://doi.org/10.1007/s00521-018-3573-3
17. Gao H, Liu X, Liu F (2018) Robust guaranteed cost control for continuous-time uncertain Markov switching singular systems with mode-dependent time delays. Neural Comput Appl. https:// doi.org/10.1007/s00521-018-3580-4

18. Xu Y, Xia X (2018) Uncertainties in the friction moment of rolling bearings based on the Bayesian theory and robust theory. Neural Comput Appl. https://doi.org/10.1007/s00521-018-3574-2

19. Xie D, Yi Y, Zhou J et al (2018) A novel temporal protein complexes identification framework based on density-distance and heuristic algorithm. Neural Comput Appl. https://doi.org/10. 1007/s00521-018-3660-5

20. Zeng F, Hu S, Xiao K (2018) Research on partial fingerprint recognition algorithm based on deep learning. Neural Comput Appl. https://doi.org/10.1007/s00521-018-3609-8

21. Ji Z, Liu W (2018) Open-circuit fault detection for three-phase inverter based on backpropagation neural network. Neural Comput Appl. https://doi.org/10.1007/s00521-018-3663-2

22. Shen C, Lin H, Guo K et al (2018) Detecting adverse drug reactions from social media based on multi-channel convolutional neural networks. Neural Comput Appl. https://doi.org/10. 1007/s00521-018-3722-8

23. Ren F, Dong Y, Wang W (2018) Emotion recognition based on physiological signals using brain asymmetry index and echo state network. Neural Comput Appl. https://doi.org/10.1007/s00521018-3664-1

24. Hu Z, Zhao Q, Wang J (2018) The prediction model of worsted yarn quality based on CNN-GRNN neural network. Neural Comput Appl. https://doi.org/10.1007/s00521-018-3723-7

25. Wang Y, Ru Y, Chai J (2018) Time series clustering based on sparse subspace clustering algorithm and its application to daily box-office data analysis. Neural Comput Appl. https://doi.org/10. 1007/s00521-018-3731-7

26. Zhai Z, Su S, Liu R et al (2018) Agent-cellular automata model for the dynamic fluctuation of EV traffic and charging demands based on machine learning algorithm. Neural Comput Appl. https://doi.org/10.1007/s00521-018-3841-2

27. Zhang Y, Li Y, Chen M (2018) Iterative learning control for linear generalized distributed parameter system. Neural Comput Appl. https://doi.org/10.1007/s00521-018-3835-0

28. Yang B (2018) Machine learning based evolution model and simulation of profit model of agricultural products logistics financing. Neural Comput Appl. https://doi.org/10.1007/s00521018-3881-7

Publisher's Note Springer Nature remains neutral with regard to jurisdictional claims in published maps and institutional affiliations. 